\title{
Diminuição da função do músculo levantador da pálpebra superior em pacientes submetidos à cirurgia de ptose palpebral involucional e dermatocálase
}

\author{
Decrease of upper eyelid levator muscle function after involutional ptosis and \\ dermatochalasis surgery
}

\author{
Eliana Forno ${ }^{1}$ \\ Eurípedes da Mota Moura ${ }^{2}$ \\ Suzana Matayoshi ${ }^{3}$ \\ Ângela Tavares Paes ${ }^{4}$ \\ Aug'usto Paranhos Junior
}

Trabalho realizado no Departamento de Oftalmologia da Faculdade de Medicina da Universidade de São Paulo - USP - São Paulo (SP) - Brasil.

Pós-graduanda (nível doutorado) da Faculdade de Medicina da Universidade de São Paulo - USP - São Paulo (SP) - Brasil.

${ }^{2}$ Doutor, Faculdade de Medicina da Universidade de São Paulo - USP - São Paulo (SP) - Brasil.

${ }^{3}$ Doutora, Médica Assistente e Chefe do Setor de Plástica Ocular do Departamento de Oftalmologia da Faculdade de Medicina da USP - São Paulo (SP) - Brasil.

${ }^{4}$ Doutor em Estatística pela USP - São Paulo (SP) Brasil.

${ }^{5}$ Livre-docente, Professor Afiliado da Universidade Federal de São Paulo - UNIFESP - São Paulo (SP) - Brasil.

Endereço para correspondência: Eliana Forno. Rua Barata Ribeiro, 490 - Cj. 38 - São Paulo (SP) CEP 01308-000

E-mail: eliana.forno@uol.com.br

Recebido para publicação em 18.05.2008

Última versão recebida em 20.10 .2008

Aprovação em 28.10.2008

\section{RESUMO}

Objetivo: Avaliar a diferença da função do músculo levantador da pálpebra superior (FMLPS), distância margem reflexo (DMR1) e altura do sulco palpebral (AS) antes e depois da cirurgia de blefaroplastia superior associada à correção de ptose palpebral. Métodos: Quarenta e quatro pacientes com blefaroptose e dermatocálase foram incluídos. Intervenção: exploração do tendão do músculo levantador da pálpebra superior (MLPS) durante a blefaroplastia, em portadores de blefaroptose e dermatocálase. Nos casos de desinserção, o tendão foi refixado ao tarso. Desfechos analisados: foram analisados de forma bilateral a diferença entre FMLPS, DMR1 e AS antes e depois da intervenção. A dependência entre os olhos foi corrigida por meio de equações de estimativa generalizada. Foi utilizada a correlação de Pearson para quantificar a dependência entre os olhos para FMLPS, DMR1 e AS. Resultados: Houve diferença significante entre as medidas de FMLPS antes e depois da cirurgia, havendo redução da excursão do MLPS após a cirurgia, diminuindo, em média, $1,1 \mathrm{~mm}(\mathrm{P}<0,001)$. As medidas pré e pós-operatória de DMR1 e AS foram estatisticamente diferentes, sendo que a DMR1 aumentou, em média, 1,6 mm e a AS diminuiu, em média, 3,97 mm ( $\mathrm{P}<0,001)$ após a cirurgia. Houve correlação significante entre os olhos para todas as variáveis propedêuticas antes e depois da cirurgia. As correlações pré-operatórias foram mais fortes no grupo sem cirurgia prévia. O mesmo foi encontrado para a correlação quanto ao grau de desinserção nos dois olhos ( $\mathrm{P}>0,01)$. Conclusão: A função do músculo levantador da pálpebra superior diminui após a cirurgia para a correção da ptose.

Descritores: Blefaroptose/cirurgia; Pálpebra/fisiopatologia; Blefaroplastia; Músculos oculomotores

\section{INTRODUÇÃO}

A ptose involucional é induzida por deiscência ou desinserção do tendão do músculo levantador da pálpebra superior (MLPS) e é caracterizada pelos seguintes sinais: sulco palpebral alto ou ausente, afinamento da pálpebra superior e função normal do MLPS (FMLPS) ${ }^{(1-8)}$. Sua freqüência foi reportada como sendo 39,1\% em brasileiros acima de 50 anos provenientes de ambulatórios de especialidades ou de enfermarias ${ }^{(9)}$.

Existem várias condições possivelmente associadas à ptose involucional, uma delas é o peso da pele excedente, cronicamente causando deis- 
cência do tendão do MLPS. Outro fator possível é a presença de cirurgia ocular prévia, como extração de catarata ${ }^{(10)}$, cirurgia para glaucoma ${ }^{(11)}$ ou mesmo cirurgia refrativa ${ }^{(12)}$. Também o trauma mecânico na pálpebra de usuários de lentes de contato ou o coçar freqüente, nos casos de alergia, podem causar desinserção do tendão do MLPS, mesmo em indivíduos jovens ${ }^{(1,13)}$.

A avaliação da FMLPS é muito importante e uma das bases para o bom planejamento cirúrgico da correção da ptose palpebral. Ela se traduz pela medida da excursão palpebral (em milímetros), partindo-se da posição extrema do olhar para baixo, até a posição extrema do olhar para cima ${ }^{(14)}$.

A FMLPS poderia, em tese, sofrer modificações após a cirurgia de correção da ptose palpebral, em decorrência do processo de cicatrização. Esta possível alteração da excursão ainda não foi abordada na literatura.

O objetivo deste estudo foi comparar as medidas obtidas na avaliação pré e pós-operatória da FMLPS, distância margem reflexo (DMR1) e altura do sulco palpebral (AS), usando um modelo estatístico de análise bilateral.

\section{MÉTODOS}

Este foi um estudo de série de casos, prospectivo, não comparativo. Os pacientes selecionados foram avaliados na clínica privada da autora (EF) e no ambulatório de Plástica Ocular do Hospital das Clínicas da Faculdade de Medicina da Universidade de São Paulo (HC-FMUSP). O estudo foi conduzido em conformidade com as resoluções nacionais e internacionais de ética em pesquisa, os pacientes assinaram termo de consentimento livre e esclarecido e o protocolo foi aprovado pelo comitê de ética em pesquisa do HC-FMUSP.

Critérios de inclusão: (1) Indivíduos acima de 45 anos, com dermatocálase e ptose palpebral involucional, de qualquer magnitude. (2) Com dermatocálase, sulco palpebral alto (acima de $12 \mathrm{~mm}$ ) e ausência de ptose. (3) Com ou sem antecedente de cirurgia ocular prévia. (4) Acuidade visual melhor ou igual a 20/200. (5) Capacidade de entender e assinar o termo de consentimento livre e esclarecido. (6) Pacientes ambulatoriais, sem necessidade de cuidados de enfermagem.

Critérios de exclusão: (1) Presença de doença ocular ativa que influencie a função palpebral. (2) Ptose de supercílio. (3) Ausência do fenômeno de Bell. (4) Olho seco clinicamente relevante. (5) Histórico de cirurgias palpebrais prévias. (6) Portadores de miopatias ou neuropatias que alterem a função palpebral. (7) Vigência de infecção ocular ativa no período de 30 dias do procedimento. (8) Histórico de alcoolismo ou de uso de drogas no período de um ano. (9) Doença psiquiátrica instável ou que necessitou de internação no último ano.

Os parâmetros analisados neste estudo foram as medidas obtidas na avaliação pré e pós-operatória da FMLPS, DMR1 e AS.

\section{Avaliação oftalmológica geral}

Os pacientes foram submetidos a exame oftalmológico completo que incluiu: acuidade visual usando tabela de Snellen (convertida para $\log \mathrm{MAR}$ ); biomicroscopia de segmento anterior; tonometria de Goldmann; biomicroscopia de segmento posterior com lente de $78 \mathrm{D}$.

\section{Avaliação da posição palpebral}

- Medida da DMR1 (medida da distância da margem palpebral superior ao centro da pupila), com o paciente em posição primária do olhar (PPO). Se a margem palpebral encobria o reflexo pupilar, a distância foi convencionalmente medida em números negativos. Considerou-se como ptose um valor de DMR1 menor ou igual a $3 \mathrm{~mm}$.

- Medida da AS com o indivíduo olhando para baixo (em $\mathrm{mm}$ ): convencionou-se medir a distância da margem até o sulco palpebral com o paciente olhando para baixo, a 45 graus.

- Medida da FMLPS: paciente em PPO, mede-se a excursão palpebral (em mm), partindo-se da posição extrema do olhar para baixo, até a posição extrema do olhar para cima, com estabilização do músculo frontal por meio de pressão digital, usando o polegar. O local para avaliação foi considerado o centro da pálpebra superior.

Todas as medidas foram efetuadas de forma manual, por meio de régua milimetrada, com auxilio de lanterna.

Para a análise estatística, os indivíduos foram divididos em dois grupos que diferiam entre si quanto ao histórico de cirurgia prévia. O propósito desta análise em subgrupos foi para excluir o viés deste fator como possível contribuinte para o grau de desinserção.

\section{Técnica cirúrgica}

O procedimento cirúrgico escolhido para a correção de todos os casos foi a abordagem anterior, assim permitindo a avaliação do tendão do MLPS e o montante de sua desinserção do tarso superior no intra-operatório e também a correção do excesso de pele e gordura herniada.

A marcação da blefaroplastia foi feita com caneta cirúrgica, iniciando-se pela determinação do sulco palpebral superior (a $10 \mathrm{~mm}$ da margem ciliar) e estendendo-se lateralmente, de acordo com o excesso de pele.

A determinação da quantidade de pele a ser removida foi feita com o paciente deitado apreendendo-se o excesso de pele com uma pinça sem dente, de modo a não everter os cílios e não provocar lagoftalmo. A marcação não ultrapassou o ponto lacrimal superior medialmente e lateralmente estendeu-se de 5 a $15 \mathrm{~mm}$ do canto externo lateral.

A anestesia local foi obtida com a infiltração de Xylocaina a 2\% (Cristália Produtos Químicos e Farmacêuticos) e Adrenalina 1:200.000 (Hypofarma), utilizando-se seringa descartável de $5 \mathrm{ml}$ e agulha $27 \mathrm{G}$, utilizando-se cerca de 3 a $4 \mathrm{ml}$ do anestésico por pálpebra, na região do subcutâneo da pálpebra superior, de maneira a distender a pele.

A incisão foi feita com bisturi de lâmina 15, iniciando-se pela marcação inferior, do canto medial ao lateral. A pele foi, então, separada do músculo orbicular subjacente, utilizandose tesoura de íris reta, sendo posteriormente excisada. Uma pequena faixa de tecido orbicular pré-septal foi removida ao 
longo de toda a pálpebra. Hemostasia foi realizada com eletrocautério bipolar e pinça delicada.

Em seguida, procedeu-se a exploração da situação do tendão do MLPS em relação ao tarso. O músculo orbicular prétarsal foi divulsionado a $10 \mathrm{~mm}$ da margem ciliar e expôs-se cuidadosamente a superfície anterior do tarso. O septo orbitário foi aberto longitudinalmente, visibilizando-se a gordura pré-aponeurótica e o referido tendão logo abaixo daquela.

Nesta etapa utilizaram-se dois afastadores de pele do tipo garra, delicados, para afastar as bordas da ferida. Verificou-se se o tendão estava ou não desinserido e, em caso afirmativo, mediu-se com compasso cirúrgico a distância do mesmo até a margem tarsal, no centro da pálpebra. A leitura da medida foi efetuada por um auxiliar que não tinha conhecimento do exame pré-operatório e foi mascarada para o cirurgião. Fotografou-se o tendão. Observar desinserção da aponeurose do levantador da placa tarsal, a distância entre as duas estruturas está delimitada pelas extremidades do compasso cirúrgico (Figura 1). Na presença de desinserção, observou-se o músculo de Müller e a conjuntiva, no espaço existente até o tarso. Procedeu-se à fixação do mesmo com fio de seda 6-0 oftalmológico, com agulha espatulada (Ethicon, Johnson \& Johnson), com pontos em "U", sendo que o primeiro foi passado na projeção do centro da pupila, com o paciente em posição primária do olhar. Foi solicitado ao paciente que abrisse a pálpebra, verificando-se a posição da mesma em relação ao eixo da pupila. Os ajustes foram feitos conforme o posicionamento obtido até que a posição palpebral fosse satisfatória. Procedeu-se às suturas complementares medial e lateral, com o mínimo de três pontos. Quando necessário, uma ou duas suturas extras foram feitas, no intuito de se obter uma curva

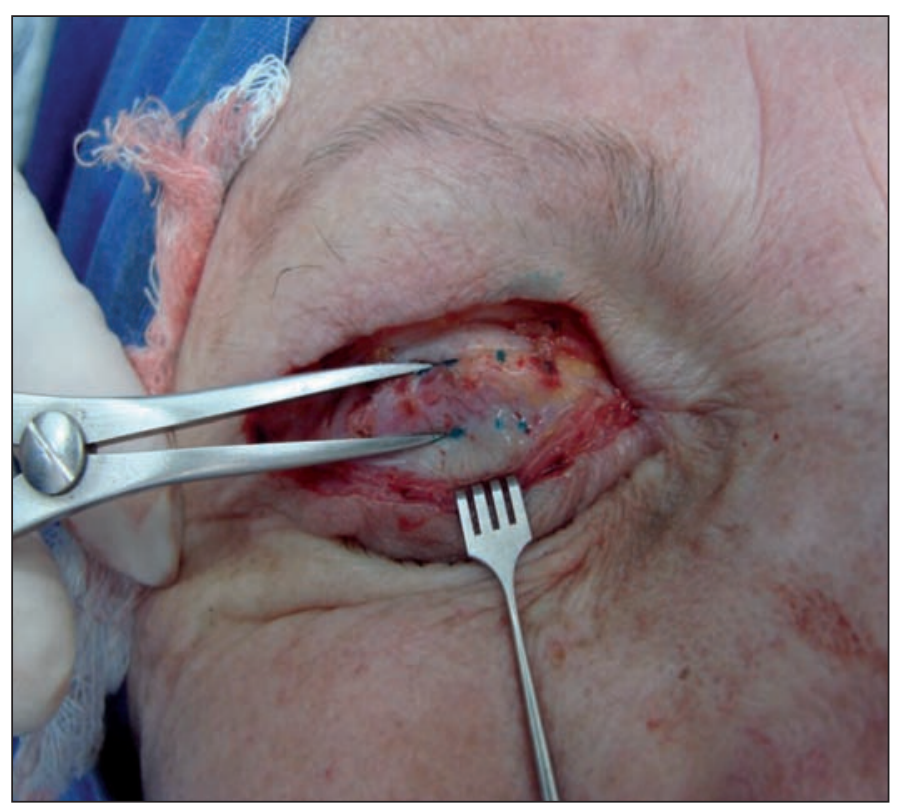

Figura 1 - Medida da distância entre a placa tarsal e a aponeurose do músculo levantador da pálpebra superior, referente a desinserção do referido músculo da placa tarsal palpebral natural e harmoniosa. A posição palpebral considerada ideal no intra-operatório foi de 1 a $2 \mathrm{~mm}$ abaixo do limbo corneal, em relação ao centro da pupila, com o paciente em PPO.

$\mathrm{O}$ excesso de gordura foi removido com cuidado, conforme a necessidade do caso. Quando não se observou desinserção, não foram realizadas as suturas de fixação do MLPS, realizando-se apenas a sutura da blefaroplastia, utilizando pontos separados de fio de nylon 6.0. Terminada a cirurgia, procedeu-se à aplicação de pomada antibiótica e compressas geladas sobre as pálpebras.

\section{Acompanhamento}

O primeiro retorno foi feito no quinto ou sexto dia pósoperatório, ocasião em que os pontos da pele foram removidos. O segundo retorno foi realizado no décimo-quarto ou décimo-quinto dia.

As medidas pós-operatórias de FMLPS, DMR1 e AS foram feitas na sexta semana após o procedimento.

Caso fosse detectado hiper ou hipocorreção no primeiro retorno, era realizada reintervenção cirúrgica precoce, entre o sétimo e décimo-quinto dia pós-operatório.

\section{Avaliação estatística}

O modelo utilizado para se avaliar a diferença entre as medidas propedêuticas (FMLPS, DMR1 e AS) pré e pósoperatórias foi o de Equações de estimativas generalizadas (GEE do inglês Generalized Estimation Equations) $)^{(15)}$ para efetuar a correção da dependência entre os olhos. A correlação entre os olhos, quanto às medidas propedêuticas nos períodos pré e pós-operatório, foi avaliada por meio da correlação de Pearson. As correlações pré-operatórias também foram efetuadas para subgrupos com e sem cirurgia prévia. Para análise estatística foi utilizado o programa SAS/STAT.

\section{RESULTADOS}

Quarenta e quatro pacientes (88 pálpebras) foram incluídas para a análise, 34 mulheres $(77,3 \%)$ e 10 homens $(22,7 \%)$. A idade variou entre 47 e 83 anos (média de $69 \pm 9,16$ anos) e o tempo de seguimento foi de 1,5 a 6 meses (média de 3,36 \pm 1,37 meses).

A estatística descritiva das medidas pré e pós-operatórias, assim como as medidas de desinserção do tendão do MLPS, estão apresentadas na tabela 1 . A desinserção do tendão foi, em média, de $5,47 \mathrm{~mm} \pm 3,02$ para o olho direito e $5,99 \mathrm{~mm} \pm 2,60$ para o olho esquerdo.

A dependência entre os olhos (correlação entre as medidas nos dois olhos) para o grau de desinserção do MLPS no peroperatório, assim como as medidas de FMLPS, DMR1 e AS no pré e pós-operatório, foram todas significativas $(\mathrm{P}>0,001)$. As correlações foram mais fortes na avaliação pré-operatória para o grupo de indivíduos não submetidos à cirurgia ocular prévia (Tabela 2). 
834 Diminuição da função do músculo levantador da pálpebra superior em pacientes submetidos à cirurgia de ptose palpebral involucional e dermatocálase

Houve diferença significante entre a FMLPS antes e depois da cirurgia, sendo que a excursão do MLPS diminui em média $1,1 \mathrm{~mm}$ (intervalo de confiança (IC) de $95 \%$ : 0,48 e 1,74, para um intercepto de 13,34) $(\mathrm{P}<0,001)$. As medidas pré e pósoperatórias de DMR1 e AS foram estatisticamente diferentes, com melhora no pós-operatório, sendo que a DMR1 aumentou, em média, 1,6 mm (IC 95\% de 3,24 e 3,70, para um intercepto de 3,47) e a AS diminuiu em média 3,97 mm (IC 95\% de 3,14 e 4,78 para um intercepto de 10,03) $(\mathrm{P}<0,001)$ (Gráfico 1).

\section{DISCUSSÃO}

No presente estudo, optou-se pela utilização das medidas tradicionais da propedêutica da ptose palpebral, usando régua milimetrada, em avaliação dinâmica da posição da margem palpebral. Embora vários autores defendam as medidas computadorizadas $^{(16-18)}$, outros ${ }^{(19)}$ descreveram boa reprodutibilidade intra e inter-observador, utilizando os métodos manuais.
Há, no entanto, relato de discordância clínica entre os métodos manuais e digitais, com o último tendendo a hipoestimar a medida de FMLPS, quando comparado ao método manual ${ }^{(17)}$. No presente estudo, todas as medidas foram efetuadas por um mesmo observador experiente, em todos os intervalos de tempo. A opção pelo uso do método manual de avaliação se deve a aproximação maior com o que existe na prática diária.

A desinserção do tendão MLPS reflete uma mudança na inserção da aponeurose, ou seja, um deslocamento para cima do ponto de transmissão da força muscular às estruturas palpebrais. Desse modo, há enfraquecimento da ação do MLPS sobre o posicionamento palpebral, com conseqüente desenvolvimento da ptose, estando a força contrátil do músculo preservada ${ }^{(20)}$.

A cirurgia corretiva restaura o ponto de transmissão de força, com melhora da abertura palpebral. Durante a cirurgia, a dissecção deve ser cuidadosa, a fim de não desinserir iatrogenicamente a aponeurose, o que pode ocorrer quando os tecidos são divulsionados partindo-se da face anterior do tarso para cima $^{(21)}$.

\begin{tabular}{|c|c|c|c|c|c|c|}
\hline & Olho & Média & Mediana & Desvio padrão & Mínimo & Máximo \\
\hline \multirow{2}{*}{ Acuidade visual (logMAR) } & OD & 0,15 & 0,10 & 0,17 & 0,00 & 0,70 \\
\hline & OE & 0,17 & 0,10 & 0,18 & 0,00 & 0,70 \\
\hline \multirow{2}{*}{ MDR1 pré* } & OD & 1,98 & 2,00 & 1,25 & 0,00 & 4,50 \\
\hline & OE & 1,75 & 2,00 & 1,22 & $-1,00$ & 4,00 \\
\hline \multirow{2}{*}{ AS pré* } & OD & 13,60 & 13,75 & 2,95 & 8,00 & 22,00 \\
\hline & OE & 14,40 & 14,75 & 2,96 & 9,00 & 20,00 \\
\hline \multirow{2}{*}{ FMLPS pré* } & OD & 14,48 & 15,00 & 1,89 & 8,00 & 20,00 \\
\hline & OE & 14,43 & 15,00 & 1,97 & 8,00 & 20,00 \\
\hline \multirow{2}{*}{ MDR1 pós* } & OD & 3,44 & 3,50 & 0,78 & 1,50 & 4,50 \\
\hline & OE & 3,50 & 3,25 & 0,84 & 1,50 & 5,00 \\
\hline \multirow{2}{*}{ AS pós* } & OD & 10,05 & 10,00 & 1,28 & 8,00 & 14,00 \\
\hline & OE & 10,02 & 10,00 & 1,17 & 8,00 & 13,00 \\
\hline \multirow{2}{*}{ FMLPS pós* } & OD & 13,36 & 14,00 & 1,89 & 8,00 & 17,00 \\
\hline & OE & 13,32 & 14,00 & 1,90 & 7,00 & 18,00 \\
\hline \multirow{2}{*}{ Desinserção do MLPS* } & OD & 5,47 & 5,75 & 3,02 & 0,00 & 11,00 \\
\hline & OE & 5,99 & 6,00 & 2,60 & 0,00 & 12,00 \\
\hline
\end{tabular}

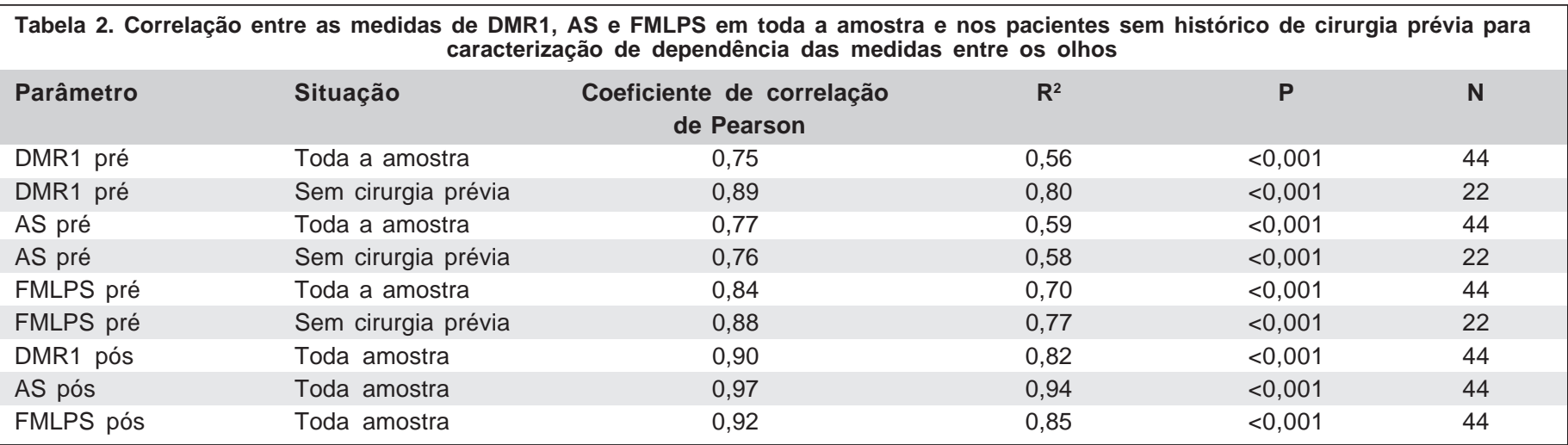




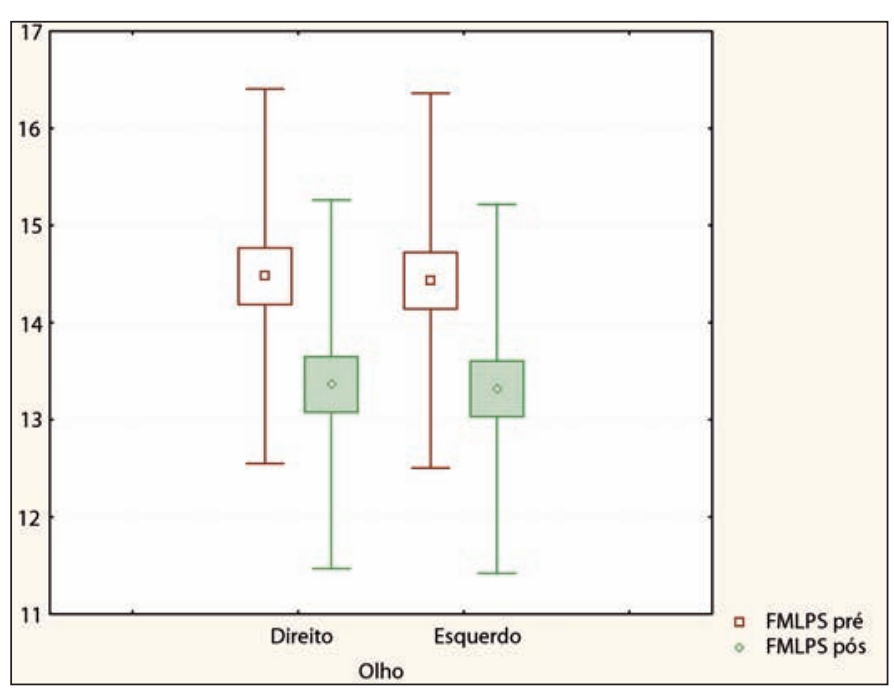

Gráfico 1 - "Box-Plot" das medidas função do músculo levantador da pálpebra superior (FMLPS) em milímetros nos dois olhos antes e depois da cirurgia (centro da caixa representa média, a caixa representa a média \pm 1 erro padrão e a linha limita \pm 1 desvio padrão)

A FMLPS é um parâmetro essencial para o planejamento de cirurgia de blefaroptose, podendo-se classificá-la como ${ }^{(22)}:$ (1) Função pobre do MLPS, quando a margem palpebral se move entre 0 e 4 mm; (2) Função moderada, de 5 a 7 mm; (3) Função boa, de 8 a 10 mm (4) Função excelente, acima de 10 mm. Na blefaroptose involucional, a FMLPS é pouco alterada ${ }^{(23-25)}$, observando-se ação média de 15,3 mm ( $\pm 1,6 \mathrm{~mm})$, com valores entre 12 e $20 \mathrm{~mm}^{(26)}$. A FMLPS média, no presente estudo, foi de $14 \mathrm{~mm}$ para ambos os olhos e, mesmo quando o valor foi baixo, esteve por volta de $8 \mathrm{~mm}$, o que ainda pode ser considerado como uma boa função. Considerou-se que o intervalo de normalidade da função é razoavelmente amplo, sendo que outros parâmetros possam influenciar a quantificação da força do $\operatorname{MLPS}^{(20)}$.

Após a cirurgia, foi observada uma limitação na FMLPS. Na ptose involucional é descrita uma diminuição da fenda palpebral na infradução, que clinicamente aparece como se houvesse um desabamento da PS quando se olha para baixo $^{(27)}$. Esse fenômeno poderia estar associado à maior elasticidade tecidual para a excursão palpebral, sendo que a refixação do tendão do MLPS, corrigindo esse fenômeno, provocaria uma diminuição da flexibilidade na excursão palpebral.

Comparando casos de blefaroplastia superior associados à conjuntivo-müllerectomia em relação a casos onde a correção da ptose foi realizada isoladamente, foi possível constatar que a elevação palpebral foi menor nos casos de cirurgia associada $^{(28)}$, provavelmente por maior fibrose e edema operatório, provocado pela reconstituição do sulco palpebral, quando o tendão do MLPS é suturado ao orbicular. A cicatrização interna da pálpebra com os tecidos externos da mesma (pele, orbicular e septo) poderia, então, ser a causa da limitação de movimento, não se tendo estudado o efeito na FMLPS.
É importante apontar que não foi realizada a sutura do tendão do MLPS no orbicular ou junto à pele e que, embora a limitação de movimento encontrada no presente estudo tenha sido estatisticamente significativa, não foi clinicamente relevante (média de $13 \mathrm{~mm}$ para os dois olhos). Como normalmente se assume que a função do MLPS é constante e não se altera com a refixação do tendão, pouca atenção tem sido dada à medida pós-operatória desse parâmetro. $\mathrm{O}$ presente estudo, entretanto, mostrou que houve redução significativa da função do MLPS. Esse achado é importante e estudos posteriores serão necessários para sua comprovação, uma vez que não existiam, até o momento, estudos que caracterizassem a função do MLPS após a cirurgia de reinserção da aponeurose do levantador.

A desinserção, por se tratar de uma característica do indivíduo, uma vez que é de origem involucional, na maioria das vezes senil, deveria ser proporcional nos dois olhos. Os resultados do presente estudo mostraram uma dependência importante entre os dois olhos para todas as medidas, o que endossa esta afirmação. As correlações entre as medidas propedêuticas foram mais fortes quando se avaliou o grupo que não tinha sido submetido a cirurgia ocular prévia. Como a cirurgia prévia é fator de risco para desinserção ${ }^{(9-11)}$, esta passaria a ser variável de confusão na avaliação da simetria das medidas entre os dois olhos do mesmo paciente. Estudos que avaliem estas variáveis devem considerar a correção de dependência, quando da realização dos cálculos estatísticos e quando do planejamento cirúrgico, fazendo mais sentido que a exploração seja bilateral.

Em conclusão, houve redução significativa da FMLPS após a cirurgia de ptose involucional, além do aumento esperado nas medidas de DMR1 e diminuição da AS. Existe uma dependência importante entre os dois olhos para as medidas propedêuticas de ptose palpebral, assim como para o montante de desinserção do tendão do MLPS.

\section{ABSTRACT}

Purpose: To evaluate the differences between upper eyelid levator muscle function (UELMF), margin reflex distance (MDR1), and eyelid crease height $(\mathrm{ECH})$ before and after ptosis and dermatochalasis surgery. Methods: Forty-four patients with blepharoptosis and dermatochalasis were enrolled. Intervention: An exploration of the levator tendon (LT) during a blepharoplasty procedure in patients with blepharoptosis and dermatochalasis and in case of its disinsertion, the tendon was reattached to the tarsus. Measured outcome: The differences between UELMF, MDR1, ECH before and after surgery were evaluated bilaterally. Dependency between both eyes was corrected by generalized estimating equations. Pearson correlation was used to evaluate the dependency of the two eyes regarding UELMF, MDR1 and ECH. Results: There was a statistical significant difference between the measures of UELMF before and after surgery with 
836 Diminuição da função do músculo levantador da pálpebra superior em pacientes submetidos à cirurgia de ptose palpebral involucional e dermatocálase

excursion decreasing by a mean value of $1.1 \mathrm{~mm}$ after the procedure $(\mathrm{P}<0.001)$. Measures of MDR1 showed an increase by a mean of $1.6 \mathrm{~mm}$ and ECH decreased by a mean of $3.97 \mathrm{~mm}$ $(\mathrm{P}<0.001)$. We found a significant correlation between both eyes for all analyzed eyelid variables, both before and after surgery. Correlation before surgery was stronger for the group without previous surgery. The same results for correlation were found for the amount of disinsertion ( $\mathrm{P}>0.001)$. Conclusions: Upper eyelid levator muscle function decreases after ptosis surgery.

Keywords: Blefaroptosis/surgery; Eyelid/physiopathology; Blefaroplasty; Oculomotor muscles

\section{REFERÊNCIAS}

1. Millay DJ, Larrabee WF Jr. Ptosis and blepharoplasty surgery. Arch Otolaryngol Head Neck Surg. 1989;115(2):198-201.

2. Jones LT, Quickert MH, Wobig JL. The cure of ptosis by aponeurotic repair. Arch Ophthalmol. 1975;93(8):629-34.

3. Morax S, Ben Ayed H, Pereira-Nunes T, Hamedani M. [Advantages of a double approach to upper blepharoplasty associated with eyelid malpositions]. J Fr Ophtalmol. 2003;26(9):993-7. French.

4. Morax S, Longueville E, Hurbli T. [Surgical treatment of myopathic ptosis. Apropos of 20 surgically treated cases]. Ann Chir Plast Esthet. 1992;37(4): 408-16. French.

5. Morax S, Herdan ML. [The aging eyelid]. Schweiz Rundsch Med Prax. 1990;79(48):1506-11. French.

6. Morax S. [Dehiscence and disinsertion of the aponeurotic bundle of the upper eyelid levator in acquired ptosis]. J Fr Ophtalmol. 1984;7(11):727-36. French.

7. Fujiwara T, Matsuo K, Kondoh S, Yuzuriha S. Etiology and pathogenesis of aponeurotic blepharoptosis. Ann Plast Surg. 2001;46(1):29-35.

8. Baker SS. Carbon dioxide laser ptosis surgery combined with blepharoplasty. Dermatol Surg. 1995;21(12):1065-70.

9. Siqueira M, Joaquim A, Schellini SA, Padovani CR, Cruz AAV. Alterações palpebrais após a idade de 50 anos. Arq Bras Oftalmol. 2005;68(3):285-90.

10. Bernardino CR, Rubin PA. Ptosis after cataract surgery. Semin Ophthalmol. 2002;17(3-4):144-8. Review.
11. Altieri M, Truscott E, Kingston AE, Bertagno R, Altieri G. Ptosis secondary to anterior segment surgery and its repair in a two-year follow-up study. Ophthalmologica. 2005;219(3):129-35.

12. Wasserman BN, Bigler B, Nipper K. Unexpected outcomes associated with laser in situ keratomileusis: ptosis, anisocoria, and "curing" of exotropia. J Cataract Refract Surg. 2005;31(6):1238-41.

13. Millay DJ. Upper lid blepharoplasty. Facial Plast Surg. 1994;10(1):18-26.

14. Gausas RE, Goldstein SM. Ptosis in the elderly patient. Int Ophthalmol Clin. 2002;42(2):61-74.

15. Liang KY, Zeger SL. Longitudinal data analysis using generalized linear models. Biometrika. 1986;73:13-22.

16. Cruz AA, Coelho RP, Baccega A, Lucchezi MC, Souza AD, Ruiz EE Digital image processing measurement of the upper eyelid contour in Graves disease and congenital blepharoptosis. Ophthalmology. 1998;105(5):913-8

17. Coombes AG, Sethi CS, Kirkpatrick WN, Waterhouse N, Kelly MH, Joshi N. A standardized digital photography system with computerized eyelid measurement analysis. Plast Reconstr Surg. 2007;120(3):647-56.

18. Edwards DT, Bartley GB, Hodge DO, Gorman CA, Bradley EA. Eyelid position measurement in Graves' ophthalmopathy: reliability of a photographic technique and comparison with a clinical technique. Ophthalmology. 2004;111(5):1029-34.Comment in: Ophthalmology. 2005;112(3):524; author reply 524-5.

19. Boboridis K, Assi A, Indar A, Bunce C, Tyers AG. Repeatability and reproducibility of upper eyelid measurements. Br J Ophthalmol. 2001;85(1):99-101.

20. Cruz AAV, Sobreira CFR, Barreira AA. Blefaroptoses. In: Cruz AAV, editor Blefaroptoses e retrações palpebrais. Rio de Janeiro: Cultura Médica; 1997. p. 36-101.

21. Martin JJ Jr, Tenzel RR. Acquired ptosis: dehiscences and disinsertions. Are they real or iatrogenic? Ophthal Plast Reconstr Surg. 1992;8(2):130-2; discussion 133.

22. Iliff JW, Pacheco EM. Ptosis surgery. In: Tassman WS, Jaeger EA, editors Duane's Clinical Ophthalmology. Philadelphia: Lippincott Williams and Wilkins; 2001. p.1-18.

23. Finsterer J. Ptosis: causes, presentation, and management. Aesthetic Plast Surg. 2003;27(3):193-204.

24. Edmonson BC, Wulc AE. Ptosis evaluation and management. Otolaryngol Clin North Am. 2005;38(5):921-46. Review.

25. Shields M, Putterman A. Blepharoptosis correction. Curr Opin Otolaryngol Head Neck Surg. 2003;11(4):261-6.

26. Frueh BR. Graves' eye disease: orbital compliance and other physical measurements. Trans Am Ophthalmol Soc. 1984;82:492-598.

27. Olson JJ, Putterman A. Loss of vertical palpebral fissure height on downgaze in acquired blepharoptosis. Arch Ophthalmol. 1995;113(10):1293-7.

28. Brown MS, Putterman AM. The effect of upper blepharoplasty on eyelid position when performed concomitantly with Muller muscle-conjunctival resection. Ophthal Plast Reconstr Surg. 2000;16(2):94-100. 